\title{
Research of Image Thresholding Segmentation Algorithm for Laser Carving
}

\author{
Jingwei Xing \\ Liren College of Yanshan University, \\ Qinhuangdao, Hebei, China \\ e-mail:xingjingwei123@163.com
}

\author{
Zhaolun Liu \\ Yanshan University \\ Qinhuangdao, Hebei, China \\ e-mail:liuzhaolun@ysu.edu.cn
}

\begin{abstract}
In the light of the major problems existing in the current laser engraving image processing software such as lack of third-dimension and lifelikeness, missing detail information, having pattern trace, this thesis using visual characteristics of the human eye, the pattern dithering method in print areas apply to the process of gray image field of laser engraving. In addition, improvement on the traditional dithering method, pattern matrix becomes homogeneous matrix by calculating, solves the problem of trace pattern, and improves the quality of carving.
\end{abstract}

Keywords- Laser Carving ; Threshold ; Tseudo-Gray ; Image processing; Digital halftoning technique

\section{INTRODUCTION}

In recent years, along with computer image processing, computer graphics etc. in the field of laser processing cross-penetration, and with significant advantages of wide processing objects, small deformation, high precision, engraving speed, ease of operation, energy saving, low pollution, remote processing, automated processing, it plays more and more essential role to improve product quality and labor productivity, achieve process automation, eliminate pollution, reduce the role of material consumption, etc. Laser engraving has huge development potential and broad market prospects [1] .

The image processing plays an important role in the laser engraving system, it will have a direct impact on image quality, speed and useful life of engraving[2-3]. But now most laser engraving stereoscopic image is not strong, it is difficult to distinguish between background and foreground, often large tracts of black or white phenomenon occurred, loss of image information severely, which causes distortion of the image [4-6]. In order to solve the above problems, the pattern dithering method and random dithering method in print areas apply to the process of gray image field of laser engraving, and proposes an improved dithering pattern, it is the better way to solve the problem of model traces and help to make the image quite real and vivid after engraving.

\section{THRESHOLDING}

Thresholding is converted grayscale image to black and white binary image, and its operation is specify a threshold value by user first, if the gray value of a pixel in the image is smaller than the threshold value, the setting value of the pixel gray is 0 , otherwise gray value is 255 . In black and white images of " 0 " represents black, "1" represents white. Engraving process is to carve out the black pixels and keep white pixels, then the image can be carved up.

\section{A. Otsu}

Otsu is currently most widely recognized and with best quality of thresholding method[7], which is to calculate a threshold value for the entire image, smaller than the threshold value is marked as object pixels (black) otherwise the background (white). The method is fast, has good quality for image with good effect especially for the image with single background; however, for image with complex background, foreground and background gray level interleaved, there will be unbearable chunk black areas, image is basically no hierarchy, no three-dimensional, its application is limited.

\section{B. Pattern dither}

Pattern dither is to use a fixed dither matrix embodiment of the original image thresholding operation. Shake table obtained from the following equation by recursive program [8-10]:

If(1), by recursion relations(2), among them, $U_{n}$ is the square of $2 n \times 2 n$, all elements of $U_{n}$ are 1 . According to this algorithm can be obtained:

$$
\begin{aligned}
M_{1} & =\left[\begin{array}{ll}
0 & 2 \\
3 & 1
\end{array}\right] \\
M_{n+1} & =\left[\begin{array}{cc}
4 * M_{n} & 4 * M_{n}+2 * U_{n} \\
4 * M_{n}+3 * U_{n} & 4 * M_{n}+U_{n}
\end{array}\right] \\
M_{2} & =\left[\begin{array}{cccc}
0 & 8 & 2 & 10 \\
12 & 4 & 14 & 6 \\
3 & 11 & 1 & 9 \\
15 & 7 & 13 & 5
\end{array}\right]
\end{aligned}
$$

Equation (3) is the standard pattern of gray 16, If the value of corresponding pixels of a gray pattern 16 is 1 , all of the corresponding points greater than 11 of ${ }^{M_{2}}$ should be output as black. Using the following method can partially avoid the above problem. Assuming artwork is 256 grayscale, use Bayer dithering table, processed as follows:

$$
\text { if }(p[y][x])>>2 \text { bayer }[y \& 7][x \& 7] \text {,then display }
$$
white point,else display black point.

$\mathrm{x}, \mathrm{y}$ represent that the pixel coordinates of the original image, $p[y][x]$ represent the point of the gradation. When displayed, make the gradation turn to the right two 
bits, into level 64 , and then do the modulo 8 arithmetic, find a corresponding point in Bayer table, compare to determine the output of the current pixel is black or white. You can see that modulo 8 arithmetic makes artwork into a corresponding one of $8 * 8$ Bayer appearances. Each point in the small comparison involved, thus avoiding the selected point and the above-mentioned division of the block is too large. Modulo 8 arithmetic is essentially incorporated as a random component .

This method can fully describe the detail of image compared with Otsu, foreground and background can be separated, has rich gradation expression of vision, but this method takes up more memory and slow down speed of output. In addition it will impose a fixed pattern on the entire image, as shown in 3-type image, has traces of the pattern after dithering.

\section{Random Dither}

In order to solve the problem with traces of pattern dither, attempt to use random dither method. The grayscale image of one pixel is converted to a halftone image of a pixel, converted into the black dots or white dots were determined by a random number, the specific method is as follows:

For each gray-scale image of a specific pixel, to produce a machine in the original grayscale image grayscale range (typically 0 to 255 ) of a random number t, and then determines the gray value of the pixel is greater than $t$, if so, then the point is converted into a pseudo gradation in the white point, otherwise converted to pseudo-gray-scale image of the black dots.

Assuming a range of gray levels from $b$ to $w$, the middle value is $m=(b+w) / 2$. For level 265 gray scale, $b=0, w=255, m=128$. If the gray scale of pixel in original is $p$, error value is error, the method is used to display the figure below the corresponding pixel value is obtained:

If $p>m$ then

display white point

error $=p-w$

else

display black point

error $=p-b$

$a \times$ error add the pixel on the right

$a \times$ error add the pixel below

$b \times$ error add the pixel on the right below

The greatest feature of the algorithm is to pass errors may occur, so that the final result of display would be close to the true value. For example, to output a 256 grayscale image, if a pixel value of the gradation is 130 , the gray scale corresponding to a gray dot. Usually gray level changes continuously, the gradation values of the adjacent pixels may be very close to the current pixel value, which occurs in and around the point may be more gray pixels. In the output of the figure, 130 is greater than 128 , it appears as a white dot, but in fact, the original 130 is a gray spot, far away from the white point 255 , error $=130-255=-125$, error is large. After added $a \times(-125)$ to the adjacent pixels, the value of adjacent pixel is close to 0 so that display the black point. Next time, error turn into a positive, so that adjacent pixels display white point, one white and one black and one white, show it just is gray. If no error passed, then shows with area. Another example of a point of the gradation is 250 , the gray scale should be a white dot, and around this point should be a white area. When the output of the drawing, although error $=-5$ is also negative, as the value is relatively small, little effect on the adjacent pixels, so it is capable of exhibiting a white area.

Image after random dither in the visual is the image of multi-gray multi-level gray scale with strong stereoscopic impression, but in fact it is the black and white dots, indicating the depth of gray levels with the density of black dots as shown in Fig .1 and Fig. .

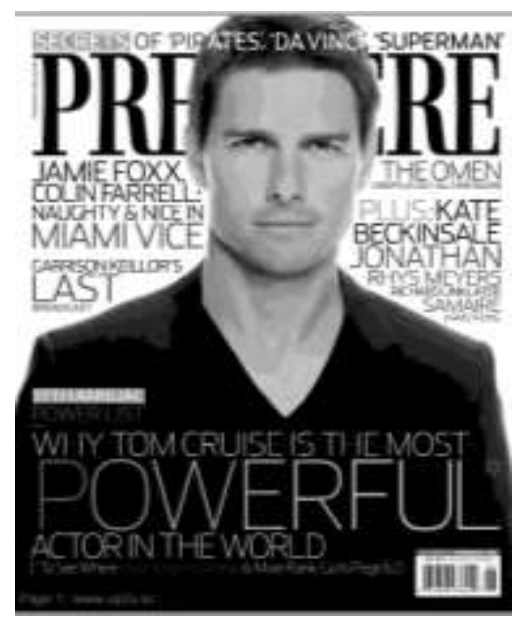

Figure 1. Original

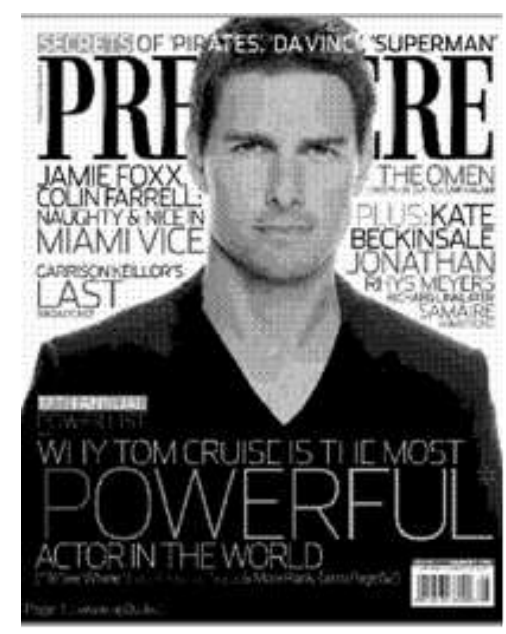

Figure 2. Image after pattern dither

Because of relatively uniformly distributed random numbers, the conversion from a random perturbation method out of the overall grayscale halftone image with the original grayscale image is closer, but also a better solution to the problem of pattern trace. Better reproduction of the original level and detail information, lifelike images with vivid. 


\section{IMPROVED PATTERN DITHER}

6、7行元素 $\left(\begin{array}{cccccccc}0 & 1 & 2 & 3 & 4 & 5 & 6 & 7 \\ 8 & 9 & 10 & 11 & 12 & 13 & 14 & 15 \\ 16 & 17 & 18 & 19 & 20 & 21 & 22 & 23 \\ 24 & 25 & 26 & 27 & 28 & 29 & 30 & 31 \\ 32 & 33 & 34 & 35 & 36 & 37 & 38 & 39 \\ 40 & 41 & 42 & 43 & 44 & 45 & 46 & 47 \\ 43 & 22 & 21 & 20 & 19 & 18 & 17 & 16 \\ 48 & 49 & 50 & 51 & 52 & 53 & 54 & 55 \\ 56 & 57 & 58 & 59 & 60 & 61 & 62 & 63\end{array}\right)$

Pattern dither uses a fixed dither matrix, it will produce significant grid-like texture.

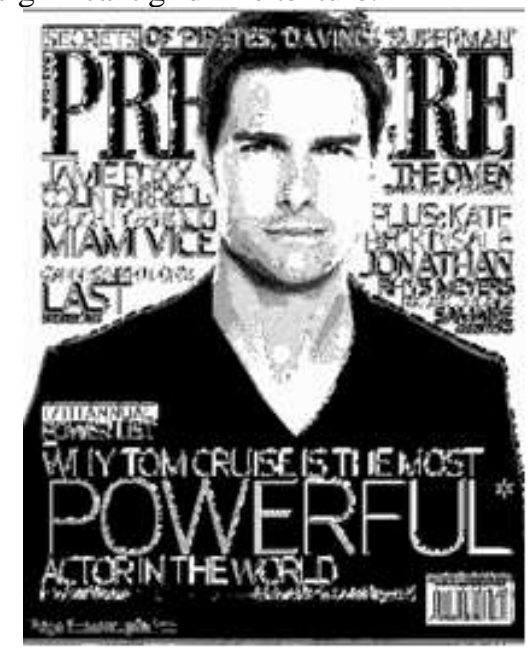

Figure 3. Improved pattern dither

From the result of random perturbation method can solve this problem, propose an improved model is now dithering method, namely the pattern dither matrix by calculating an arbitrary construct any column or row elements and elements and are equal.

$8 * 8$ matrix is uniformly distributed in space, can ensure that the image of the local and global homogeneity, so the image processing is completed as shown in Fig .3, substantially no traces of the template image distinct, clear gray level performance, effect is ideal.

\section{CONCLUSIONS}

So far, the threshold segmentation method has been proposed is far more than that, the number of method could not be counted. Because of different methods applied to different objects, it is difficult to make unified criteria. To achieve better effect on image of laser engraving, the original image need to be preprocessing, usually contrast enhancement, and edge sharpening, and then smoothed. Through several experiments, especially for the good quality of the image is a single background image can be used for image processing Otsu; random dither, pattern dither fundamentally broke through the traditional method, combined with the characteristics of human vision and laser error inherent characteristics of sculpture, with a density of black pixels represent that portion of the image in grayscale, which satisfy the detailed description, high-layering, rich gradation requirements, also a better solution to the problem of model traces; the pattern matrix transformation into a uniform matrix method, not only absorbed the random perturbation of the overall uniformity, but also to avoid the randomness of random dither in the local area, so strong stereoscopic image post-processing, structured, there are detailed descriptions, gray degree level rich, basically no pattern traces.

In the laser engraving system, which consists of image processing, poor fill control, draw vector graphics, output control, the use of above method for complex image processing could achieve the expected requirements, but should be noted that the area of the relative complex image cannot be too small, the spot size is generally $0.5 \sim 1 \mathrm{~mm}$ when using laser engraving, sometimes it will cause a multi-point coincidence, detailed description is not accurate enough. Therefore, in the actual operation to be combined with the specific characteristics and requirements of engraving engraved image of the object to select the appropriate image processing method.

\section{Acknowledgements}

It is a project supported by the National Natural Science foundation, China (NO.61307110) and the Qinhuangdao Municipal Science and Technology Research and Development Program, China (NO.201401A016)

\section{References}

[1] M.Takahiro ; F.Kenshi; Y.Takaji; W.Mitsuo. "Fabrication of finely pitched lyso arrays using subsurface laser engraving technique with picosecond and nanosecond pulse lasers." IEEE Transactions on Nuclear Science, v 61, n 2, p 1032-1038, April 2014

[2] M.Takahiro ; F.Kenshi . "Development of PET detectors using monolithic scintillation crystals processed with sub-surface laser engraving technique". IEEE Transactions on Nuclear Science, v 57, n 5 PART 1, p 2455-2459, October 2010

[3] Z. Bao;H.Beinan;Z. Lei; Z.Tianyi "The 3D laser engraving pavilion design based on ergonomics" Applied Mechanics and Materials, v 224, p 312-315, 2012, Industrial Design and Mechanical Power

[4] H. William C.J.; M.Robert S.; M.Lawrence; M. Wendy; L.Thomas K. "Light-sharing interface for dMiCE detectors using sub-surface laser engraving" IEEE Nuclear Science Symposium Conference Record, 2013, 2013 IEEE Nuclear Science Symposium and Medical Imaging Conference, NSS/MIC 2013

[5] S.Q. Zhang; D.M.Zhang;Q.H. Feng. "Research on the key technology of laser engraved image"Advanced Materials Research, v 989-994, p 3919-3921, 2014, Materials Science, Computer and Information Technology

[6] Y. Eiji ; H.Yoshiyuki; T.Hideaki; "The X'tal cube PET detector with a monolithic crystal processed by the $3 \mathrm{D}$ sub-surface laser 
engraving technique: Performance comparison with glued crystal elements"Nuclear Instruments and Methods in Physics Research, Section A: Accelerators, Spectrometers, Detectors and Associated Equipment, v 723, p 83-88, 2013

[7] S. Joon-Hong; L. Ju-Jang; Lee, D.Hyun; K. Hee-Shin; S.Hyonkee . "Laser micro-patterning software development for manufacturing of pattern roll for printed electronics" IEEE International Symposium on Industrial Electronics, 2013, 2013 IEEE International Symposium on Industrial Electronics, ISIE 2013

[8] Z.X.Gong ;J.C. Cheng; J.H.Shen, “A block mapping binarizing algorithm capable of partialy reserving intensity distribution of original image"Proceedings - 4th International Congress on Image and Signal Processing, CISP 2011, v 3, p 1194-1196, 2011, Proceedings - 4th International Congress on Image and Signal Processing, CISP 2011

[9] S.Saloomeh ; J.Laurent; S.François-Xavier; M.Benoit; "Rando mly driven Fuzzy key extraction of unclonable images"Proceedings - International Conference on Image Processing, ICIP, p 4329-4332, 2010, 2010 IEEE International Conference on Image Processing, ICIP 2010 - Proceedings

[10] Y.H.Chen;J.N.,Lu. "Making plates the digital way"Advanced Materials Research, v 479-481, p 561-564, 2012, Advanced Mechanical Design 